\title{
EM-Lens Enhanced Power Transfer and Multi-Node Data Transmission for Implantable Medical Devices
}

\author{
Peilong Feng ${ }^{* \dagger}$, Michal Maslik*†, and Timothy G. Constandinou*† \\ *Department of Electrical and Electronic Engineering, Imperial College London, SW7 2BT, UK \\ ${ }^{\dagger}$ Centre for Bio-Inspired Technology, Institute of Biomedical Engineering, Imperial College London, SW7 2AZ, UK \\ Email: \{peilong.feng14, michal.maslik12, t.constandinou\}@imperial.ac.uk
}

\begin{abstract}
This paper presents a robust EM-lens-enhanced wireless power transmission system and a novel multiple-node data communication method for distributed implantable medical devices. The proposed techniques can solve the common issues caused by multiple implanted devices, such as low power transfer efficiency through biological tissues, uneven delivered power for distributed devices and interference between simultaneous wireless power and data transmission. A prototype system has been manufactured with discrete components on FR4 substrate as a proof of concept. The EM-Lens-enhanced inductive links can expand the power coverage of transmitting ( $T \mathbf{x})$ coil from $9 \mathrm{~mm} \times 5 \mathrm{~mm}$ to $14 \mathrm{~mm} \times 13 \mathrm{~mm}$, and double the recovered $\mathrm{DC}$ voltage from $1.8 \mathrm{~V}$ to $3.2 \mathrm{~V}$ at $12.5 \mathrm{~mm}$ distance. Data communication is achieved by novel low-power back-scattering CDMA scheme. This permits transmission of data from several nodes all operating with the same carrier frequency simultaneously reflecting the power carriers to the primary side. In this paper, we demonstrate simultaneous communication between two nodes at $125 \mathrm{kbps}$ with $1.05 \mathrm{~mW}$ power consumption.
\end{abstract}

\section{INTRODUCTION}

The recent interest in development of distributed implantable medical devices has brought up new demand for research of novel wireless information and power transmission (WIPT) systems. Those have to facilitate wireless power transfer (WPT) efficiently through biological tissues while ensuring extensive and uniform power coverage for distributed implants alongside robust power supply delivery for freepositioned devices. In addition, wireless information transmission (WIT) method has to be devised making use of minimal power consumption while providing sufficient bandwidth for simultaneous recovery of data from multiple implants.

This is commonly solved by a three-coil inductive WPT with extra resonator to boost the power transfer efficiency (PTE) while extending the area of power coverage for the entire network of implants [1]. A possible alternative solution is unfocused ultrasonic WIPT. This has been shown to wirelessly power multiple implants in the peripheral nervous system at $45 \mathrm{~mm}$ depth or in the central nervous system through thinned skull [2].

However, while partially solving the problem of delivering power to implant networks, those solutions are incapable of solving misalignment issues between the transmitter ( $\mathrm{Tx}$ ) and the receiver ( $\mathrm{Rx}$ ) due to positional or angular deviation, especially in the critical state of perpendicular alignment, where the power transmission efficiency approaches zero. Re-

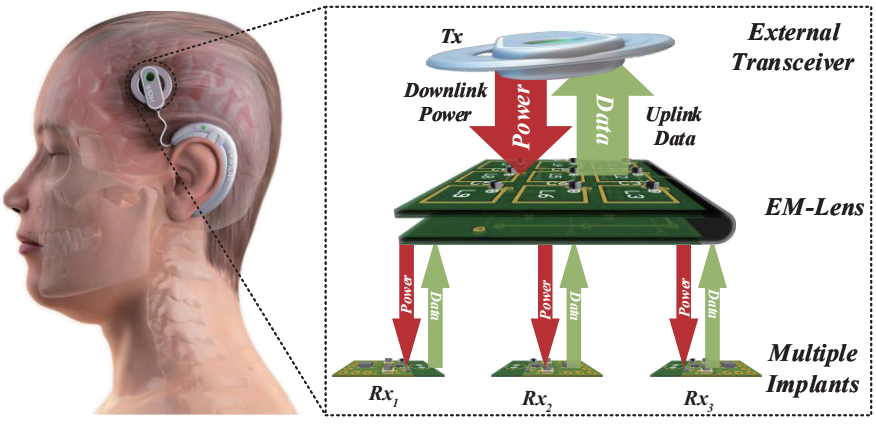

Fig. 1. Proposed architecture of EM-Lens enhanced wireless power transfer and multiple nodes data communication system for implantable medical devices.

cently, artificial metamaterials (EM-Lens) have been verified to improve the near-field wireless power transfer efficiency and reduce the impact of positional and angular offset [3], [4].

WIT schemes for implantable medical devices typically rely on communication techniques used in cellular phone networks [5], [6] such as time division multiple access (TDMA) and frequency division multiple access (FDMA) which both support simultaneous transmission from several devices. They are however limited by the interference between the wireless power carrier and data communication as well as insufficient bandwidth for hundreds or thousands of implanted devices in addition to difficulties associated with assigning time or frequency slots.

As an alternative, we therefore consider the use of Codedivision multiple access (CDMA). This allows for communication of multiple nodes at the same time transmitting at the same frequency by employing orthogonal-code modulation. The frequency-spreading property

To overcome forementioned difficulties, this paper proposes an enhanced wireless power transmission system through artificial EM-lens and a simultaneous multiple-node data communication technique among distributed neural sensors, shown in Fig 1. The rest of this paper is organised as follows: Section II introduces the design concept and system architecture; Section III details the operating principle of the proposed WIPT; Section IV shows the measured results of the preliminary print circuit board (PCB) prototype, and Section V and VI reveals the advantages and disadvantages of the current system then points out the future direction of the next generation wireless 
infrastructure.

\section{System OVERVIEW}

The system concept of the EM-lens enhanced brain-machine interface and the simplified system diagram are shown in Fig 1. The WPT system establishes on EM-Lens enhanced inductive coupling. The external transceiver generates and amplifies the $433 \mathrm{MHz}$ power carrier and transmits to the artificial EM-Lens that is composed of repeated geometric patterns to modify in the propagation and distribution of electromagnetic fields [7]. The electromagnetic field is relayed through the artificial EMLens to the underneath distributed neural implants, thereby achieving the strong electromagnetic coupling and extending the energy coverage. Then, the neural implants convert the induced electromagnetic energy into a DC supply voltage by a power conversion and management subsystem to load neural recorders or stimulators.

The data communication technique bases on backscatter modulation and CDMA. The data generated by each implanted devices is encoded by orthogonal channelisation codes and simultaneously sent to the external transceiver by backscattering incoming power carrier. The external receiver recovers the baseband information from all nodes, then the data from each node is decoded by using specific channelisation codes. The advantages of this proposed WIPT approach can be summarised as follows.

- The range and uniformity of power coverage is extended while improving power transmission efficiency, and the power variations caused by positional and angular deviation can be relieved, compared to conventional 2-coil inductive link;

- The Backscatter-CDMA coding technique benefits from low power consumption since no data carriers need to be generated in implanted devices. It also increases the channel capacity of the communication system and avoids the intermodulation among different frequency bands.

\section{WIPT OPERATING PRINCIPLE}

We briefly describe the operating principle of the proposed system as Power in, Data out, which means to wirelessly send power into the distributed implants as downlink and receive the data generated by the multiple implants outside the body as uplink. We show the system schematic in Fig 2. The external device comprises transceiver, power amplifier (PA), Tx coil $L_{1}$, and $\mathrm{Rx}$ antenna $A_{1}$. The external device transmits power while demodulates the received signal. The receiving system contains a microcontroller (MCU), digitally tuned capacitors, Rx coil $L_{4}$, and frequency tuning capacitors $C_{T_{D} a t a}$. This system allows frequency adjustment to maximise power transfer efficiency. Also, the backscatter modulation and CDMA encoder are implemented on the microcontroller. The following subsections describe the operating principle of WPT and multiple-node WIT.

\section{A. EM-Lens Enhanced Wireless Power Transfer System}

Metamaterials (EM-Lens) enhanced wireless energy transfer for inductive links has been extensively investigated [3],

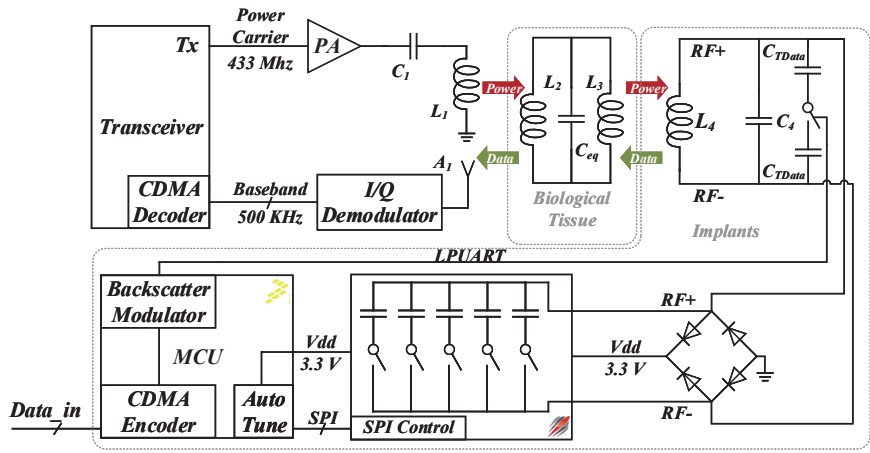

Fig. 2. Simplified block diagram of external devices, implanted wireless interface and equivalent circuit model of EM-Lens.

[4]. It is common to use a split-ring resonator (SRR) as a unit that arranged in a specific order, such that the entire resonator exhibits a negative magnetic permeability, enhancing the coupling coefficient of the inductive link. The arrangement of the resonators here is based on our previous design and simulation [7], and fabricated on the FR4, which improves the energy transmission efficiency and reduces the deviation of energy coverage, and also helps to increase the distance of wireless energy transmission.

\section{B. Multiple-Node Backscatter-CDMA Data Communication}

CDMA technology has been widely adopted in the mobile communication system, which benefits from its large capacity and excellent anti-interference performance. In this paper, multi-channel concurrent data transmission is realised through the spread spectrum communication technology, then transmit the digital signal by utilising low power backscatter. The specific method is to encode the communication data by using the 16-element Walsh matrix as channel vectors and then send the encoded information to the switch of the tuning capacitor $C_{T \text { Data }}$ through the LPUART of the microcontroller to vary the resonance frequency of secondary LC tank and scattering the power carriers back to the primary side. The external receiving device uses inherent I/Q demodulation hardware to remove the power carriers in the reflected signal, and then individually decodes each channel using the pre-defined channel vectors.

\section{PROOF OF CONCEPT}

To prove the feasibility of the proposed system, we utilise discrete components and FR4 substrate to implement the entire system and verify the transmission efficiency of the wireless power system and the functionalities of the multiple-node information communication system.

\section{A. Wireless Power Transfer Verification}

The experiment setup for the wireless power transfer system is shown in Fig 3. The $433 \mathrm{MHz}$ power carrier is generated by the RF signal generator SML03 (Rohde \& Schwarz, Germany) and then transmitted to the Tx coil through the power amplifier HMC1099 (Analog Devices, USA). The PCB-based EMLens is coated in the biologically compatible PMMA and 

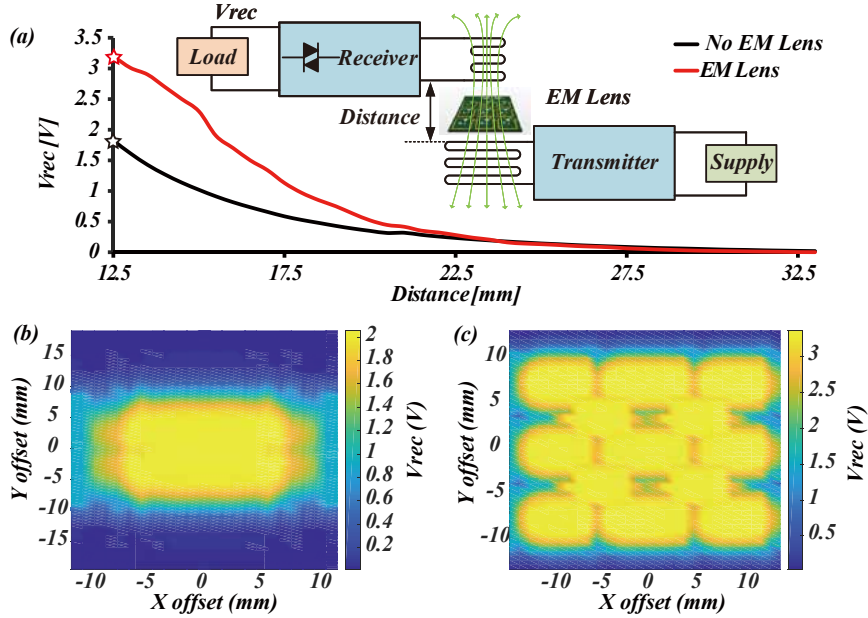

Fig. 3. Measured results of EM-Lens enhanced WPT: (a)Rectified voltage on Rx side with various distance, compared with air gap inductive link; (b) Power coverage of air gap inductive link; (c) Power coverage of EM-Lens enhanced inductive link.

placed between the Tx and Rx coils to extend the coverage of electromagnetic fields. The $\mathrm{Rx}$ coil is connected to the Schottky diodes JDH2S02SL (Toshiba, Japan) for full-bridge rectification. The rectified DC voltage is conveyed to the load resistor. The power supply voltage is probed by using LeCroy 7300A oscilloscope (Teledyne Technologies, USA) through the Hirose U.FL RF connector (Hirose Electric, Japan). Two RVX500 manual linear motion manipulators (Wentworth Laboratories, USA) hold the Tx and Rx coils. Thus, the relative position between these coils can be precisely adjusted. This experimental setup is used to measure the supply voltage variation from $12.5 \mathrm{~mm}$ to $32.5 \mathrm{~mm}$ distance in the air gap, and within $\pm 13 \mathrm{~mm}$ of parallel offset, also compared to a wireless power transfer system without EM-Lens enhancement. According to the measured results in Fig 3, the advantages of EM-Lens enhanced inductive power transfer system can be summarised as: 1) Within $20 \mathrm{~mm}$ of the air gap, EM-Lens has a significant improvement on the received supply voltage; 2 ) EM-Lens can increase the energy coverage by four times from the previous $9 \mathrm{~mm} \times 5 \mathrm{~mm}$ to current $14 \mathrm{~mm} \times 13 \mathrm{~mm}$.

Before implementing the EM lens into the wireless power transfer system, the most critical task is to guarantee that the self-resonant frequency of the entire electromagnetic induction system is consistent. The resonance based inductive link can significantly enhance power transfer efficiency [8]. The Tx coil, EM lens, and Rx coil are based on our previous studies [7], [9] with matching and tuning capacitors to ensure that return loss of each coil is less than $-10 \mathrm{~dB}$ at $433 \mathrm{MHz}$, which ensures that most of the electromagnetic power can pass through the inductor networks smoothly. According to Fig 4, the return losses of the Tx coil, EM-Lens and the unloaded $\mathrm{Rx}$ coil are $-11.52 \mathrm{~dB},-7.93 \mathrm{~dB}$ and $-10.95 \mathrm{~dB}$, respectively.

\section{B. Backscatter-CDMA Data Transmission Verification}

The experimental setup of the wireless data transmission system is shown in Fig 6. The software-defined radio frequency (SDR) platform comprises the signal transceiver

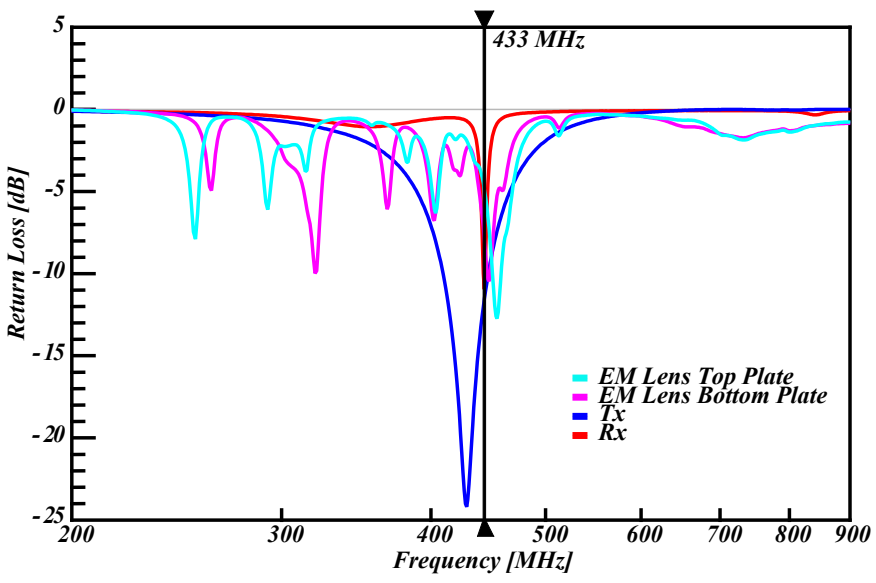

Fig. 4. Measured results of return loss about Tx, EM-Lens and Rx.

daughter board (AD-FMCOMMS4-BZ,Analog Devices, USA) and an FPGA motherboard (Zedboard, Avnet, USA) through a high-speed FMC connector. We take advantage of the programmable logic on the FPGA to implement two necessary functions. The first one is the digital signal process chain including automatic gain controller, raised cosine FIR filter, and CDMA decoder, after converting the backscattered signals received by the antenna to baseband through the built-in I/Q demodulation circuitry in the transceiver. The second one generates a $433 \mathrm{MHz}$ digital signal to configure the transmitting power carrier. Then this carrier is amplified by PA and transmits to the primary LC tank. On the secondary side, the MCU (MKL03Z32VFG4, NXP, Netherlands) is used to control the digitally tunable capacitor (PE64102, Peregrine Semiconductor, USA) to tune the resonance frequency of the secondary LC tank through the SPI bus and adjust the supply voltage.

The MCU uses a 4-bit $\times 4$-bit Walsh matrix as the channelisation codes to encode the sending data by using XOR logic. Then encoded data controls the RF switch (RV1C002UN, Rohm semiconductor, Japan) through the LPUART of MCU at $500 \mathrm{kHz}$. This switch in series with a $5 \mathrm{pF}$ tuning capacitor can add or disconnect from the secondary LC tank, resulting in alteration of its resonant frequency. The value of the capacitor for backscatter is 2.7 times of the unit capacitor for supply voltage adjustment which generates a noticeable variation of $\approx 500 \mathrm{mV}$ on primary differential $\mathrm{RF}+/$ - signal. In addition, a $660 \mathrm{pF}$ smoothing capacitor at the output of rectifier limits these RF variation within $40 \mathrm{mV}$ on the rectified supply voltage. The external transceiver detects the amplitude difference of $433 \mathrm{MHz}$ power carrier by using I/Q demodulation to converts the modulated data signal to the baseband. Then it digitalises the analog baseband signal through a 12-bit ADC, transfers to a constant level with adaptively gain control, and reduces intersymbol interference through raised cosine filtering. The demodulated signal at this stage is shown in Fig 5. Subsequently, each nodes information is decoded with a predefined correlation 4-bit Walsh matrix. The brief description of the decoding process is to multiply the demodulated signal by using each channelisation codes 


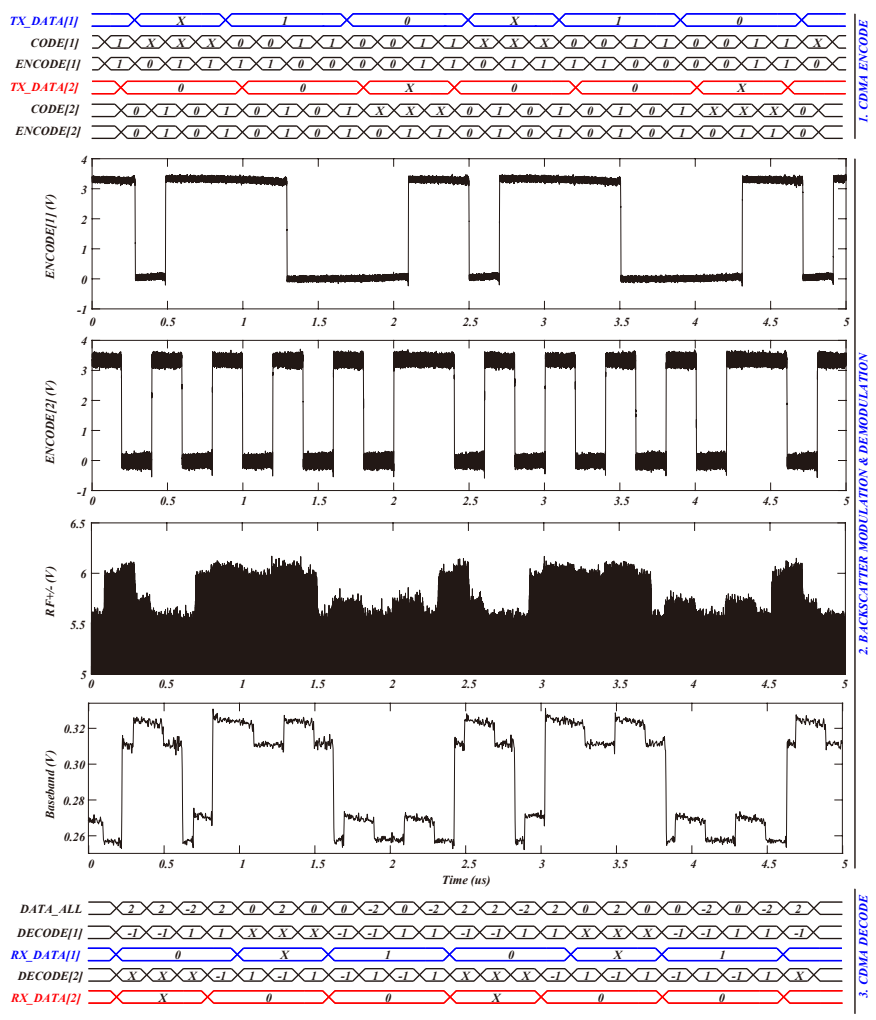

Fig. 5. Time diagram of 2-node backscatter-CDMA data communication contains: (1). CDMA encoding stage; (2). Backscatter modulation and demodulation; (3). CDMA decoding process.

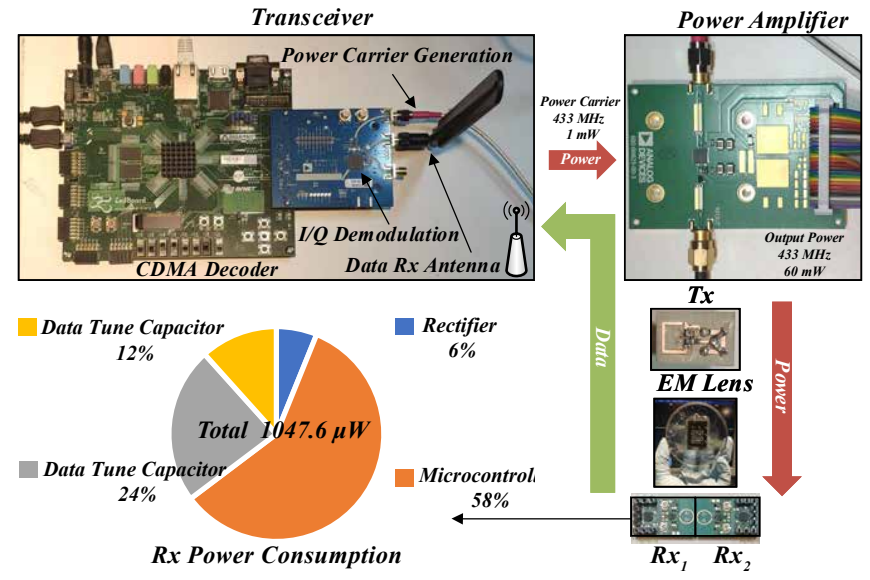

Fig. 6. Experiment setup and Rx power consumption breakdown.

and then average the decoded signal during every four clock cycle to restore original information sent by each node. The power breakdown of entire Rx system is shown in Fig 6 and each Rx device consumes 1047.6 $\mu \mathrm{W}$.

\section{Discussion}

The experimental results validate the theoretical principles presented in section IV, and the presented PCB prototype to verify the power coverage of EM-lens, and achieve data communication between two nodes. The EM-Lens can be extended to provide greater power coverage to hundreds of distributed devices because it is composed of the repetitive

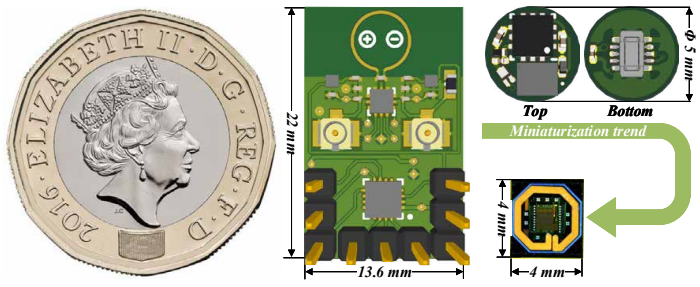

Fig. 7. Future direction of wireless interface for implantable devices.

resonant pattern. Also, EM-lens are preferred to fabricate on a flexible biocompatible substrate [10], which fit on the curvature of the brain and reduce the risk of the bodys rejection. In addition, we have scaled distributed Rx devices down to $5 \mathrm{~mm} \times 5 \mathrm{~mm}$ on circular FR4 substrates, which offer possibilities for next-stage in-vivo experiments with rodents.

\section{CONCLUSION}

This work demonstrates that EM-Lens enhanced wireless power transfer system to solves the issues of low power transfer efficiency for implantable medical devices and alleviate the sensitivity of positional offset and angular offset resulting in insufficient received supply energy. The proposed multiuser communication method achieves data communication among multiple node and avoids interference between the power and data transmission. Backscatter modulation is realized by adopting power carriers to modulate sending data, and CDMA is used to spread spectrum so that multiple node can simultaneously communicate at the same frequency. The proposed technique of wireless power transmission and data communication shows a new direction for future research about completely wireless infrastructure for distributed $\mathrm{mm}$ sized neural implants.

\section{ACKNOWLEDGEMENT}

This work was supported by the Engineering and Physical Sciences Research Council (EPSRC) grant EP/M020975/1.

\section{REFERENCES}

[1] S. A. Mirbozorgi et al., "Robust wireless power transmission to $\mathrm{mm}$ sized free-floating distributed implants," IEEE TBioCAS, vol. 11, no. 3, pp. 692-702, June 2017.

[2] M. M. Ghanbari et al., "A sub-mm ultrasonic free-floating implant for multi-mote neural recording," arXiv, 2019.

[3] R. A. Shelby et al., "Experimental verification of a negative index of refraction," Science, vol. 292, no. 5514, pp. 77-79, 2001.

[4] B. Wang et al., "Wireless power transfer: Metamaterials and array of coupled resonators," Proceedings of the IEEE, vol. 101, no. 6, pp. 13591368, June 2013.

[5] J. Lee et al., "Wireless power and data link for ensembles of sub-mm scale implantable sensors near 1ghz," in BioCAS2018, 2018, pp. 1-4.

[6] M. M. Ghanbari et al., "A sub-mm ultrasonic free-floating implant for multi-mote neural recording," arXiv, 2019.

[7] P. Feng et al., "Robust wireless power transfer to multiple mm-scale freely-positioned neural implants," in BioCAS2018, Oct 2018, pp. 1-4.

[8] A. Kurs et al., "Wireless power transfer via strongly coupled magnetic resonances," Science, vol. 317, no. 5834, pp. 83-86, 2007.

[9] P. Feng et al., "Chip-scale coils for millimeter-sized bio-implants," IEEE TBioCAS, vol. 12, no. 5, pp. 1088-1099, Oct 2018.

[10] M. O. de Beeck et al., "Ultra-thin biocompatible implantable chip for bidirectional communication with peripheral nerves," in BioCAS2017, Oct 2017, pp. 1-4. 